\title{
Time-resolved studies of energy transfer in thin films of green and red fluorescent proteins
}

\author{
J. M. Zajac, ${ }^{1}$ M. Schubert, ${ }^{1}$ T. Roland, ${ }^{1}$ C.-M. Keum, ${ }^{1}$ I. D. W. Samuel, ${ }^{1}$ and \\ M. C. Gather ${ }^{1}$ \\ Organic Semiconductor Centre, SUPA, School of Physics and Astronomy, \\ University of St Andrews, St Andrews, Fife KY16 9SS, \\ United Kingdom
}

Biologically derived fluorescent proteins are attractive candidates for lasing and sensing due to their excellent optical properties, including their high quantum yield, spectral tunability and robustness against concentration quenching. Here, we report a time-resolved study of the fluorescence dynamics of thin films of Enhanced Green Fluorescent Protein (EGFP), the red-emitting tandem-dimer protein tdTomato and blends of EGFP and tdTomato. We characterized the exciton dynamics by using spectrally and time-resolved measurements of fluorescence and observed a threefold reduction in lifetime when going from solution to thin film, down to $1 \mathrm{~ns}$ and $0.6 \mathrm{~ns}$ for EGFP and tdTomato, respectively. This finding is attributed to a dipole-dipole non-radiative Förster resonant energy transfer (FRET) in solid-state. We also studied the temporal characteristics of FRET in blended thin films and found increased non-radiative transfer rates. Finally, we report efficient sensitisation of a semiconductor surface with a protein thin film. Such a configuration may have important implications for energy harvesting in hybrid organic-inorganic solar cells and other hybrid optoelectronic devices.

Keywords: photoluminescence, protein, time correlated single-photon counting, streak camera, energy transfer 


\section{INTRODUCTION}

Green Fluorescent Protein (GFP) is a highly fluorescent macromolecule that occurs naturally in the jellyfish Aequorea victoria ${ }^{1}$. Multi-billion year evolution has led to remarkable optical properties of GFP, such as a high photoluminescence quantum yield (PLQY, $\eta_{\mathrm{PLQY}} \approx 0.6-0.8$, depending on exact type). Over the past two decades, the development of relatively simple genetic methods to express GFP in a wide variety of different organisms has revolutionised many areas of biology, including in vivo microscopy and gene expression studies ${ }^{2}$. This has been greatly aided by the development of numerous mutants of the wild-type GFP. Today, these mutants cover the whole visible spectrum and in some cases offer additional functionality, such as reversible fluorescence photo-switching or sensing capabilities $^{3}$. The Enhanced Green Fluorescent Protein (EGFP) is a monomeric mutant of wild-type GFP, i.e., it has a dramatically reduced tendency to spontaneously form dimers of two protein molecules. Like wild-type GFP, it also emits in the green region of the visible spectrum (with an emission peak at $510 \mathrm{~nm}$ ) but offers increased photo-stability and brightness. This makes EGFP one of the most widely used GFP mutants and renders it a useful model system for photo-physical studies. tdTomato is another widely used fluorescent protein variant. It occurs in a tandem dimer configuration and emits in the red region of the visible spectrum (with a peak emission wavelength of $590 \mathrm{~nm})^{4}$. Very recently, thin films of neat fluorescent protein were used as the active material in vertical-cavity surface-emitting lasers (VCSEL) operating in both the photonic and the polaritonic regime ${ }^{5,6}$.

Unlike many synthetic organic dyes, fluorescent proteins derived from GFP show relatively weak fluorescence quenching in densely packed films, which has been attributed to their 3-dimensional conformation ${ }^{7}$. The GFP molecule consists of a characteristic amino acid nano-cylinder with a diameter of $2.4 \mathrm{~nm}$ and a height of $4.2 \mathrm{~nm}$ (a so-called $\beta$-barrel, Fig. 1a), which holds the emitting fluorophore at its centre ${ }^{8,9}$. The $\beta$-barrel thus acts as a protective cylinder or 'bumper' that separates fluorophores from each other and protects them from the environment.

Time-resolved spectroscopy can provide deep insights into the photophysical processes implicated in the emission of a material. For fluorescent proteins, a better understanding of their photophysics may not only pave the way to using biologically produced proteins in next-generation photonic devices but can also teach us important lessons on bio-inspired 
design of more efficient emitters. The spectral and temporal properties of the emission from solutions of fluorescent proteins have been studied extensively ${ }^{10}$. Recently, it was found that while the PLQY of fluorescent proteins is also very high in the solid state, it is lower than in solution ${ }^{7}$, which indicates that these materials can potentially be further optimized once the exact photophysics are known. However, there is limited information on the temporal dynamics of thin films, with the exception of a very recent study on thin films of EGFP ${ }^{11}$ and an older report that used multifrequency phase fluorimetric data to determine the fluorescence lifetime of crystals of wild-type $\mathrm{GFP}^{12}$.

Here, we fill this gap and report on detailed time- and spectrally resolved emission measurements on thin films of EGFP, tdTomato and blends thereof. Our study uses streak camera measurements and time-correlated single photon counting (TCSPC) to reveal the time-scales of the exciton dynamics in these films. We find clear evidence of a strong resonant energy transfer in films made of protein blends. Moreover, we discuss non-radiative energy transfer from protein films to bulk layers of an inorganic semiconductor (i.e., between an EGFP film and a silicon wafer). Our findings will help to guide the way to newly designed synthetic fluorescent proteins and will enable the development of new biocompatible lasers. Hybrid protein-semiconductor structures may develop into a new paradigm for the sensitization of silicon ${ }^{13,14}$, here with the protein as a sensitiser that absorbs incident light and then transfers its energy to silicon. This is relevant to applications in solar cells, photo-diodes, and transistors.

\section{ENERGY DIAGRAM, ABSORPTION AND PHOTOLUMINESCENCE SPECTRA}

To characterise the absorbance and photoluminescence (PL) of fluorescent protein samples, we prepared low-concentration solutions $\left(c=10^{-2} \mathrm{mM}\right)$ and also fabricated thin films of fluorescent protein by drop-casting highly concentrated solutions $(c=1 \mathrm{mM})$ on glass substrates. The concentrations of the solutions discussed in this manuscript were deduced directly from the maximum absorbance of the proteins (i.e., absorbance at $490 \mathrm{~nm}$ for EGFP and $555 \mathrm{~nm}$ for tdTomato) and thus represent the concentrations of the optically active fluorophores (see Experimental).

Fig. 1a shows a possible energy digram of the EGFP molecule, with protonated states A 
and deprotonated states B and I, following the scheme proposed by Cotlet et al. ${ }^{15}$. In brief, State $A_{1}$ absorbs at $400 \mathrm{~nm}$ leading to $A_{1}^{*}$, which emits at $450 \mathrm{~nm}$. In solution, $A_{1}^{*}$ has a lifetime of approximately $250 \mathrm{ps}$ and can also undergo a fast conformational change into $A_{2}^{*}$, which then mostly relaxes through excited state proton-transfer into the deprotonated state $I^{*}$. Also in solution, state I absorbs at $490 \mathrm{~nm}$ and emits around $509 \mathrm{~nm}$ with a lifetime of approximately $3.5 \mathrm{~ns}$, whereas state B absorbs at $470 \mathrm{~nm}$ and emits above $509 \mathrm{~nm}$ with a lifetime of 2.9 ns. As will be evidenced and explained in more detail below, the excited state lifetimes for thin films are significantly reduced, to $180 \mathrm{ps}$ for state $A_{1}^{*}$ and $1.1 \mathrm{~ns}$ for states $I^{*}$ and $B^{*}$.

Fig. 1b shows the absorption and PL spectra of an EGFP solution and a thin film of EGFP. The absorption spectra can be decomposed into three Gaussian peaks, corresponding to state A, absorbing at $400 \mathrm{~nm}$, and states B and I, absorbing at $470 \mathrm{~nm}$ and $490 \mathrm{~nm}$, respectively (shown for the thin film spectrum in Fig. 1b) ${ }^{15}$. The PL spectrum has a maximum at $510 \mathrm{~nm}$ with an extended shoulder at $540 \mathrm{~nm}$, corresponding to emission from excited states B and I (excitation wavelength, $\lambda_{\text {exc }}=400 \mathrm{~nm}$ ). The thin film spectra are qualitatively similar to those seen in solution, exhibiting generally the same absorption and emission maxima. The PLQY for the EGFP film was measured to be approximately 0.3, i.e., about a $50 \%$ reduction compared to the literature-value for EGFP in solution ${ }^{3}$. While the thin films studied here were completely dried, earlier work has found that keeping the protein films moist modifies their properties and can substantially increase their brightness and presumably their PLQY ${ }^{6,7}$.

The absorption and PL spectra of a tdTomato solution and a thin film of tdTomato are shown in Fig. 1c. For the solution, the absorption maximum occurs at a wavelength of $555 \mathrm{~nm}$, and the peak of the PL spectrum is found at $585 \mathrm{~nm}\left(\lambda_{\text {exc }}=400 \mathrm{~nm}\right)$. Interestingly, for the dense film of tdTomato, the absorption spectrum shows an additional shoulder at $580 \mathrm{~nm}$ and an extended long-wavelength tail. The PL of the thin film is strongly redshifted compared to that of the solution, with a peak at $630 \mathrm{~nm}$. Because films made from lower-concentration solutions showed no red-shift ${ }^{7}$, we attribute this observation to a reabsorption effect. (The drop-casting process used for film preparation leads to a coffeestain type topography of the film and thus only to partial coverage of the substrate ${ }^{7}$. The absorption spectra shown in Fig. 1c therefore strongly underestimate the absolute absorbance of the film.) The PLQY for the film was 0.1, which is considerably lower than the value of 
0.7 reported for tdTomato solutions ${ }^{4}$ and which indicates that tdTomato is more prone to concentration quenching than EGFP.

\section{TIME-RESOLVED EMISSION CHARACTERISTICS}

To map the time dynamics of excitons in thin films of fluorescent proteins, we combined streak camera and TCSPC measurements. Fig. 2a shows the spectrally and time-resolved emission from a thin film of EGFP under excitation with a femtosecond laser and recorded with a streak camera. Qualitatively, one can see a very fast fluorescence decay for wavelengths $<490 \mathrm{~nm}$ and a longer decay, stretching out over about $1 \mathrm{~ns}$, for longer wavelengths. The fluorescence decay for two $20 \mathrm{~nm}$ wide spectral bands located around 450 and $510 \mathrm{~nm}$, respectively, is shown in Fig. 2b. At $450 \mathrm{~nm}$ the $1 / e$ temporal width of the emission is $180 \mathrm{ps}$ which is consistent with but somewhat shorter than the 250 ps lifetime observed for the $A^{*}$ state emission in earlier work ${ }^{15}$. The decay of emission at $510 \mathrm{~nm}$ has a $1 / e$ width of 1140 ps. We attribute the emission in this band to a combination of emission from the $I^{*}$ and $B^{*}$ states but note that for the solid-state protein film the emission decay of these states is about three-times faster than in the solution case studied earlier. This indicates an increase in non-radiative energy transfer to quenching sites, which will be discussed in more detail later. In an attempt to determine the solid-state lifetimes of the $A^{*}, I^{*}$ and $B^{*}$ states separately, we have also performed global tri-exponential fits to the data (results not shown). However, while this reliably identified a fast decay component in the blue range of the spectrum $(\leq 490 \mathrm{~nm})$ that is likely associated with emission from the $A^{*}$ state, it was not possible to confidently separate decay components in bands located at wavelengths $>509$ and $<509 \mathrm{~nm}$ and thus to distinguish the lifetimes of the $I^{*}$ and the $B^{*}$ states as was done for the solution case in Refs 15 and 16.

Next, we discuss our TCSPC experiments in which we were able to obtain fluorescence decay data over a much longer measurement window compared to the streak camera measurement above. In Fig. 3, we compare the PL decay curves of solutions and thin films of EGFP and tdTomato. Fig. 3a shows the fluorescence decay at $510 \mathrm{~nm}$ for a $1 \mathrm{mM}$ solution of EGFP as well as the instrument response function (IRF) of the TCSPC system. Using a procedure analogous to Ref. 15, we performed a tri-exponential fit to the data that uses deconvolution of the IRF to improve the temporal resolution of our measurement to about 
100 ps while maintaining a long measurement window. The lifetimes extracted from this fit were $0.3,2.9$ and $3.5 \mathrm{~ns}$, with 1:100:100 relative amplitudes. From our streak camera measurements above and from time-resolved emission spectra measurements on EGFP solutions in Ref. 15, no substantial contribution of a short sub-ns decay component is expected in the $510 \mathrm{~nm}$ band. The extracted lifetimes are in good agreement with previously reported values, i.e. 2.8 and $3.3 \mathrm{~ns}$ for wild-type $\mathrm{GFP}^{16}$ and $0.25,2.7$ and $3.4 \mathrm{~ns}$ for $\mathrm{EGFP}^{15}$, respectively.

To simplify the notation in the following discussion, we introduce an average lifetime, which is given by

$$
\tau_{a v}=\frac{A_{1} \tau_{1}+A_{2} \tau_{2}+A_{3} \tau_{3}}{A_{1}+A_{2}+A_{3}}
$$

where $A_{i}$ and $\tau_{i}$ refer to the amplitude and lifetime of the three exponential decay components. Compared to the solution, the PL decay for a thin film of EGFP is considerably shorter (compare Fig. 3a and c; $\tau_{a v}=3.5$ versus $1 \mathrm{~ns}$ ). This reduction in lifetime is consistent with our previous streak camera measurement.

Like for EGFP, the PL decay for tdTomato is also considerably reduced in the thin film, with extracted average lifetimes of 3.4 and $0.6 \mathrm{~ns}$ for solution and thin film, respectively.

Fig. 3e compares the average lifetime extracted from TCSPC measurements on EGFP and tdTomato solutions with a range of different concentrations (between $10^{-4}$ and $2 \mathrm{mM}$ ) as well as for thin films of EGFP and tdTomato, which have nominal concentrations of $\approx 40-50 \mathrm{mM}$. The average lifetime remains approximately constant at $\tau_{a v} \approx 3.5 \mathrm{~ns}$ for the investigated range of concentrations but then drops for the thin films as described above.

This observation is consistent with a previous report ${ }^{7}$, where the intermolecular distance between EGFP molecules at which FRET becomes efficient was estimated to $4.6 \mathrm{~nm}$, i.e., slightly higher than the average intermolecular separation in solid state films, which has been previously estimated to $R=3.3 \mathrm{~nm}$.

Another contribution to the reduced lifetime may be due to reduced excited state proton transfer. Proton transfer is likely mediated by water molecules that are either encaged in the $\beta$-barrel structure of the protein molecule ${ }^{8}$ or attached to water binding sites on the outside of the $\beta$-sheets forming the EGFP cylinder ${ }^{10}$. In solution, there are sufficient water molecules to support an efficient transfer. However, when the film dries, water evaporates, and thus the excited state proton transfer to the emissive B and I states is slowed down. This explanation is consistent with recent results on thin films with different water contents ${ }^{11}$. (The difference in the absolute lifetime values between Ref. 11 and our work is likely due to 
differences in sample fabrication.)

Finally, for comparison, we also measured the PL lifetimes for a synthetic pyrromethene dye (PM556, Exciton Inc.). This dye has spectral properties similar to EGFP, but in a thin film its PL is so heavily quenched by non-radiative relaxation that it was not possible to determine a fluorescence lifetime for solid-state PM556. By contrast, such a catastrophic concentration quenching is absent in thin films of fluorescent proteins from the GFP family.

\section{ENERGY TRANSFER IN PROTEIN BLENDS}

Next, we studied thin films made of a blend of EGFP and tdTomato (7:3 EGFP to tdTomato by weight; corresponding to an approximate 7:3 ratio of EGFP to tdTomato fluorophores because the molecular weight of tdTomato is approximately four times that of EGFP and tdTomato is a tetramer whereas EGFP is a monomer). Fig. 4a shows a PL spectrum of an EGFP thin film, together with an absorption spectrum of a tdTomato thin film. There is a significant spectral overlap, indicating that efficient FRET between EGFP (energy donor) and tdTomato (energy acceptor) can be achieved. Fig. 4b shows the PL spectrum of a film of the protein blend. Two distinct peaks are observed, corresponding to the EGFP emission maximum at $510 \mathrm{~nm}$ and the tdTomato emission maximum at $630 \mathrm{~nm}$. Although the number of tdTomato fluorophores in the film is substantially smaller than the number of EGFP fluorophores, its emission is five times more intense than the emission from EGFP.

Fig. 4c compares PL decay curves at the EGFP peak emission wavelength $(510 \mathrm{~nm})$ for a pure EGFP thin film and for the EGFP:tdTomato blend film. The extracted average lifetime for the blend is $\tau=0.6 \mathrm{~ns}$, which represents a $40 \%$ reduction compared to the pure EGFP. By contrast, the average lifetime around the peak emission wavelength of tdTomato $(625 \mathrm{~nm})$ is nearly identical for the blend and a pure tdTomato film (Fig. 4d). Together these two observations indicate that efficient FRET occurs from EGFP to tdTomato in the blended film. 


\section{ENERGY TRANSFER BETWEEN PROTEIN FILMS AND SEMICONDUCTORS}

Finally, we explored if fluorescent proteins can mediate energy transfer to inorganic semiconductors like silicon. One of the limiting factors for the performance of silicon based solar cells and other silicon based optoelectronic devices is its relatively low absorption. Silicon sensitisation via non-radiative energy transfer is a promising avenue to increase its effective absorption ${ }^{17}$. Depositing a monolayer of a sensitiser with an effective band gap that exceeds the bandgap of silicon can induce an efficient energy transfer process. To date, this has been achieved by using sensitisers made from dyes ${ }^{18}$ and colloidal quantum dots ${ }^{13,14}$. Here, we explore the use of biologically derived fluorescent proteins for this purpose.

Fig. 5a shows a schematic illustration of the sample structure used in this experiment. An $\mathrm{Al}_{2} \mathrm{O}_{3}$ spacer layer with variable thickness was deposited on a silicon wafer using atomic layer deposition (ALD). The nominal thicknesses of the spacer layer were $0,3,5,10,15,30$, and $50 \mathrm{~nm}$ and these were confirmed to be accurate to within $\pm 5 \%$ using ellipsometry. On top of the spacer, a thin layer of EGFP was deposited using drop casting from a low-concentration solution $\left(c=10^{-3} \mathrm{mM}\right)$. Fig. 5b shows the PL spectra recorded from substrates with different spacer layer thicknesses (the acquisition conditions were identical for all samples). While the shape of the PL spectrum was mostly unaffected by the thickness of the spacer, the intensity reduced dramatically as the thickness of the spacer layer was reduced from $30 \mathrm{~nm}$ to $0 \mathrm{~nm}$ (i.e., for the bare $\mathrm{Si}$ substrate).

We analysed the PL data following the procedure described in Ref. 19, plotting $\left(A_{0} / A-1\right)$ as a function of the spacer thickness (Fig. 5c). Here, $A$ refers to the spectrally integrated fluorescence across the entire emission band and $A_{0}$ corresponds to the fluorescence emission in the absence of the silicon surface. In our case, the $A_{0}$ value was obtained from the sample with a $30 \mathrm{~nm}$ thick spacer layer. For comparison, we also measured the PL for thicker spacer layers but did not observe any additional changes in intensity (Fig. 5b). ( $\left.A_{0} / A-1\right)$ is directly proportional to the ratio between the non-radiative energy transfer rate to $\mathrm{Si}$ and the radiative rate of neat EGFP (i.e., $k_{E T} / k_{R}$ ). Our results indicate a decrease in the fluorescence intensity of $98 \%$ between the sample with the $30 \mathrm{~nm}$ spacer layer and the bare Si substrate. Such a strong fluorescence quenching is consistent with non-radiative energy transfer to silicon. Based on the model of dipole-dipole interaction, a distance dependency 
of $\left(A_{0} / A-1\right)$ in the form of $d^{-3}$ is expected, but there are clear deviations from this in the data. Therefore, the current results cannot be explained simply based on a single-dipolesemiconductor model of energy transfer. A similar behaviour was described in Ref. 18 and references therein. Fig. 5c also shows an empirical fit with an exponential decay function, i.e., $c \times \exp (-\gamma d)-y_{0}$ which describes the data better than the $d^{-3}$ fit.

\section{SUMMARY}

In summary, we have characterised the exciton dynamics in thin films of the fluorescent proteins EGFP and tdTomato. While we observed a threefold reduction in the characteristic decay times between films and solutions, fluorescent proteins importantly maintain bright fluorescence even in neat solid-state films. We believe that the reduction in fluorescence lifetime is related to the size of proteins from the GFP family, which are generally smaller than their Förster radius, and may also result from structural changes associated with the loss of water molecules around or within the protein structure. In the future, newly designed synthetic proteins, such as RNA aptamers ${ }^{22,23}$, may allow fine-tuning of protein size and fluorophore structure such that they are less prone to drying effects and thus offer even better PLQY in the solid state. We have also characterised the energy transfer in films made of protein blends and observed rapid donor accept transfer. Finally, we demonstrated an efficient energy transfer between protein film and a semiconductor that resulted in a $98 \%$ quenching of PL, thus opening up the possibility of developing hybrid inorganic-protein devices.

\section{ACKNOWLEDGEMENTS}

JMZ thanks P. Andreakou for discussion. The authors thanks K. Ostermann (TU Dresden) for protein expression. This research was financially supported by the European Research Council of the European Union (ERC Grant Agreements No. 640012/ABLASE and 321305/EXCITON), by the Scottish Funding Council (through SUPA), by EPSRC (through the CDT Capital Equipment funding stream, EP/L017008/1) and by a European Commission Marie Curie Career Integration Grant (PCIG12-GA-2012-334407). MS acknowledges funding by the European Commission through a Marie Sklodowska-Curie Individual Fellow- 
ship (659213). IDWS acknowledges support from a Royal Society Wolfson Research Merit Award.

\section{EXPERIMENTAL SECTION}

\section{A. Protein preparation}

The open reading frames of EGFP and tdTomato were amplified with PCR and cloned into vector pET23b (Novagen) using standard molecular biology methods. The corresponding protein contains an N-terminal hemagglutinin tag for immune detection and a His6 tag for protein purification. The Escherichia coli strain BL21(DE3)pLysS (Novagen) was transformed with the construct using LB medium with ampicillin (100 $\mathrm{mg} / \mathrm{mL})$ and chloramphenicol $(34 \mathrm{mg} / \mathrm{ml})$ for selection. Cells were grown at $30{ }^{\circ} \mathrm{C}$ until an OD600 (optical density at $600 \mathrm{~nm}$ ) of 0.5 to 0.6 was reached and were harvested 4 hours after protein expression was induced with isopropyl-b-D-thiogalactopyranoside (final concentration, $0.5 \mathrm{mM}$ ). The cells were resuspended in buffer $[20 \mathrm{mM}$ tris $(\mathrm{pH} 7.9), 500 \mathrm{mM} \mathrm{NaCl}$, and $5 \mathrm{mM}$ imidazole] and disrupted by sonification and treatment with lysozyme, and the target protein was purified using His-Bind Resin (Novagen). After elution, the protein-containing fraction was dialyzed against phosphate-buffered saline twice using a Slider-A-Lyzer Dialysis cassette (10K molecular weight cutoff, $15 \mathrm{ml}$ ) (Thermo Fisher Scientific). Subsequently, the protein

was filtered and centrifuged to remove buffer salts and increase the protein concentration (to up to $350 \mathrm{~g} / \mathrm{L}$ ). This solution was later diluted with water to obtain desirable concentrations.

To prepare the blend samples, we used stock solutions of the pure proteins and mixed them at a 7:3 (EGFP:tdTomato) ratio by weight. Thin films were then formed by drop casting onto glass substrates.

\section{B. Absorption measurements}

The absorbance of protein solutions and films was determined with a Cary 300 Bio spectrophotometer. To convert absorbance into concentration, the used molar absorbance and molar mass were $\epsilon=55,000 \mathrm{M}^{-1} \mathrm{~cm}^{-1}$ and $m=27 \mathrm{kDa}$ for EGFP and $\epsilon=138,000 \mathrm{M}^{-1} \mathrm{~cm}^{-1}$ and $m=112 \mathrm{kDa}$ for tdTomato, respectively ${ }^{20,21}$. 


\section{PL measurements}

For excitation and emission measurements, a steady-state fluorimeter was used. The PLQY of thin protein films was measured using an integrating sphere setup (Hamamatsu). High-resolution temporal measurements were made using a streak camera (Hamamatsu C10910-05) with a time resolution down to $3.5 \mathrm{ps}$ and an overall time window of approx.

$2 \mathrm{~ns}$; samples were excited using 200 fs laser pulses at $5 \mathrm{kHz}$ repetition rate generated by optical parametric amplifier pumped by a Pharos regenerative amplifier from Light Conversion. For other time-resolved measurements, a fluorimeter with a TCSPC module was used (Edinburgh Instruments) and samples were excited with a single-mode laser emitting at approx. $400 \mathrm{~nm}$. The temporal IRF was $600 \mathrm{ps}$, which after deconvolution resulted in a time resolution of $100 \mathrm{ps}$. For low-concentration solutions (between 0.1 and $0.01 \mathrm{mM}$ ), we used cuvettes with an optical path length of $10 \mathrm{~mm}$. For higher concentrations, we used cuvettes with a reduced optical path length, down to $100 \mu \mathrm{m}$, to avoid reabsorption effects.

\section{REFERENCES}

${ }^{1}$ O. Shimomura, FEBS Letters 104, 220 (1979).

${ }^{2}$ M. Chalfie, Y. Tu, G. Euskirchen, W. W. Ward, and D. C. Prasher, Science 263, 802-805 (1994).

${ }^{3}$ R. Y. Tsien, Annu. Rev. Biochem. 67, 509-544 (1998).

${ }^{4}$ N. C. Shaner, P. A. Steinbach, and R. Y. Tsien, Nat. Methods 2, 905-909 (2005).

${ }^{5}$ C. P. Dietrich, S. Höfling, and M. C. Gather, Appl. Phys. Lett. 105, 233702 (2014).

${ }^{6}$ C. P. Dietrich, A. Steude, L. Tropf, M. Schubert, N. M. Kronenberg, K. Ostermann, S. Höfling, and M. C. Gather, Sci. Adv. 2, e1600666 (2016).

${ }^{7}$ M. C. Gather and S. H. Yun, Nat. Commun. 5, 5722 (2014).

${ }^{8}$ M. Ormo, A. B. Cubitt, K. Kalio, L. A. Gross, R. Y. Tsien, and S. J. Remingtont, Science 273, 1392-1395 (1996).

${ }^{9}$ J. J. Thor, Chem. Soc. Rev. 38, 2935 (2009).

${ }^{10}$ M. Chalfie and S. R. Kain, eds., Green Fluorescent Protein: Properties, Applications and Protocols (Wiley, 2005). 
${ }^{11}$ C. P. Dietrich, M. Siegert, S. Betzold, J. Ohmer, U. Fischer, and S. Höfling, Appl. Phys. Lett. 110, 043703 (2017).

${ }^{12}$ M. A. Perozzo, K. B. Ward, R. B. Thompson, and W. W. Ward, J. Biol. Chem. 263, 7713-7716 (1988).

${ }^{13}$ S. Chanyawadee, R. T. Harley, M. Henini, D. V. Talapin, and P. G. Lagoudakis, Phys. Rev. Lett. 102, 077402 (2009).

${ }^{14}$ P. Andreakou, M. Brossard, C. Li, M. Bernechea, G. Konstantatos, and P. G. Lagoudakis, EPJ Web of Conferences (2013).

${ }^{15}$ M. Cotlet, J. Hofkens, M. Maus, T. Gensch, M. Van der Auweraer, J. D. G. Michiels, V. G. Maarte, J. Vanderleyden, A. J. W. G. Visser, and F. C. De Schryve, J. Phys. Chem. B 105, 4999-5006 (2001).

${ }^{16}$ G. Striker, V. Subramaniam, C. A. M. Seidel, and A. Volkmer, J. Phys. Chem. B 103, 8612 (1999).

${ }^{17}$ D. Dexter, J. Lumin. 18/19, 779-784 (1979).

${ }^{18}$ L. Danos, R. Greef, and T. Markvart, Thin Solid Films 516, 7251-7255 (2008).

${ }^{19}$ T. Hayashi, T. Castner, and R. Boyd, Chem. Phys. Lett. 94, 461-466 (1983).

${ }^{20}$ N. C. Shaner, M. Z. Lin, M. R. McKeown, P. A. Steinbach, K. L. Hazelwood, M. W. Davidson, and R. Y. Tsien, Nat. Methods 5, 545-551 (2008).

${ }^{21}$ R. E. Campbell, O. Tour, A. E. Palmer, P. A. Steinbach, G. S. Baird, D. A. Zacharias, and R. Y. Tsien, Proc Natl Acad Sci USA 99, 7877-7882 (2002).

${ }^{22}$ J. S. Paige, K. Y. Wu, and S. R. Jaffrey, Science 333, 642-646 (2011).

${ }^{23}$ R. J. Trachman III, L. Truong, and A. R. Ferre-D'Amare, Trends Pharmacol. Sci. 38, 928-939 (2017). 

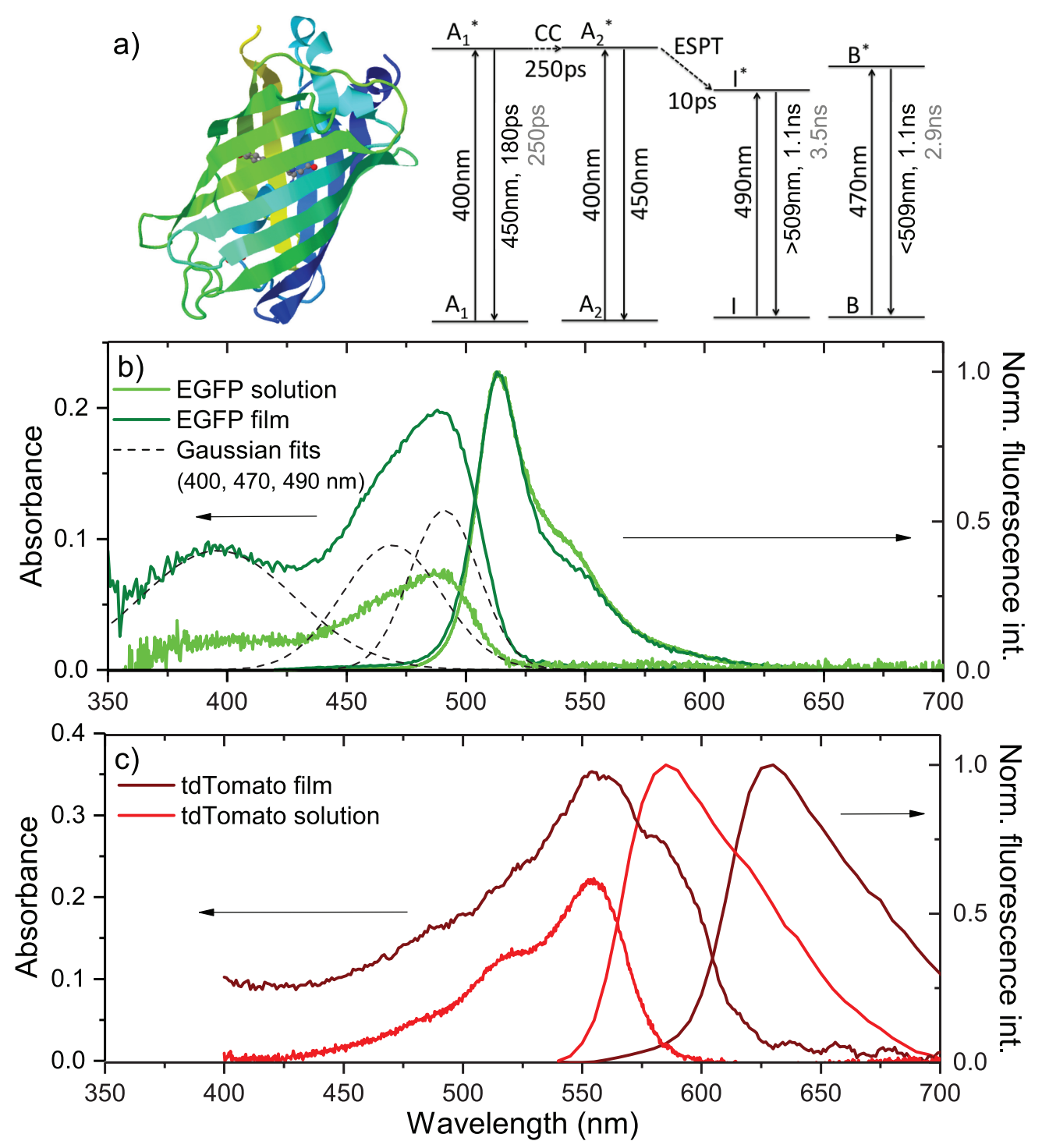

FIG. 1. a) Illustration of the molecular structure of the fluorescent protein EGFP with the fluorophore enclosed by a $\beta$-barrel structure. A possible energy diagram of EGFP, with three states of the fluorophore, a protonated state $\mathrm{A}$ and deprotonated states $\mathrm{B}$ and I. In the diagram, CC denotes conformational change, and ESPT denotes excited-state proton transfer. The energies and lifetimes of the different radiative transitions are indicated, with lifetimes in black for thin films and lifetimes in grey for solutions. b) Absorption and emission spectra of a solution and a thin film of EGFP. The absorption spectra were decomposed into three Gaussian peaks, corresponding to states A, absorbing at $400 \mathrm{~nm}, \mathrm{~B}$, absorbing at $470 \mathrm{~nm}$, and I, absorbing at $490 \mathrm{~nm}$. c) Absorption and emission spectra of a solution and a thin film of tdTomato. 

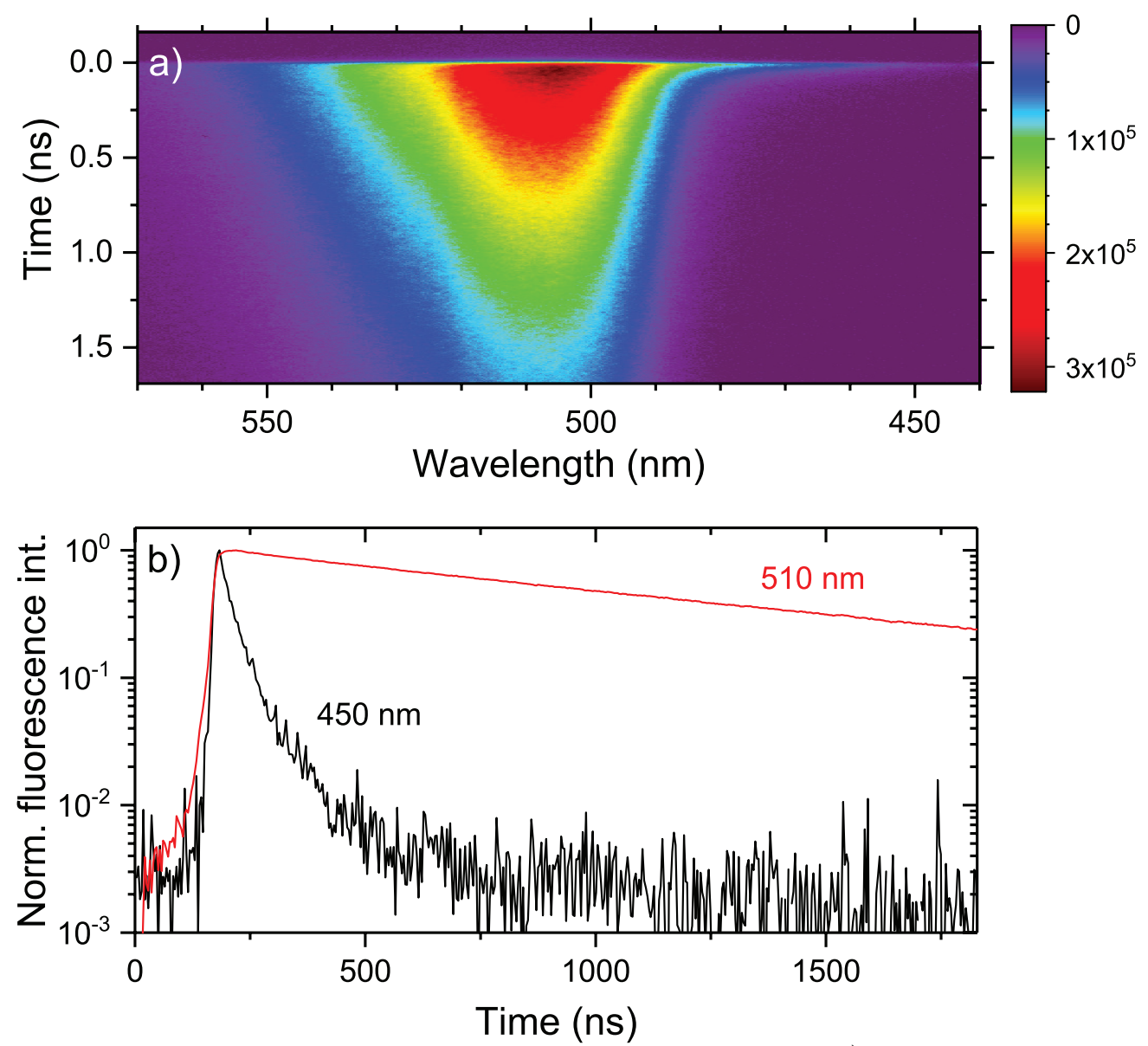

FIG. 2. Time-resolved exciton dynamics for a thin film of EGFP. a) Spectrally and time-resolved PL measurement obtained by a streak camera. b) Fluorescence decay data extracted from panel a for two $20 \mathrm{~nm}$ wide spectral bands around 450 and $510 \mathrm{~nm}$. 

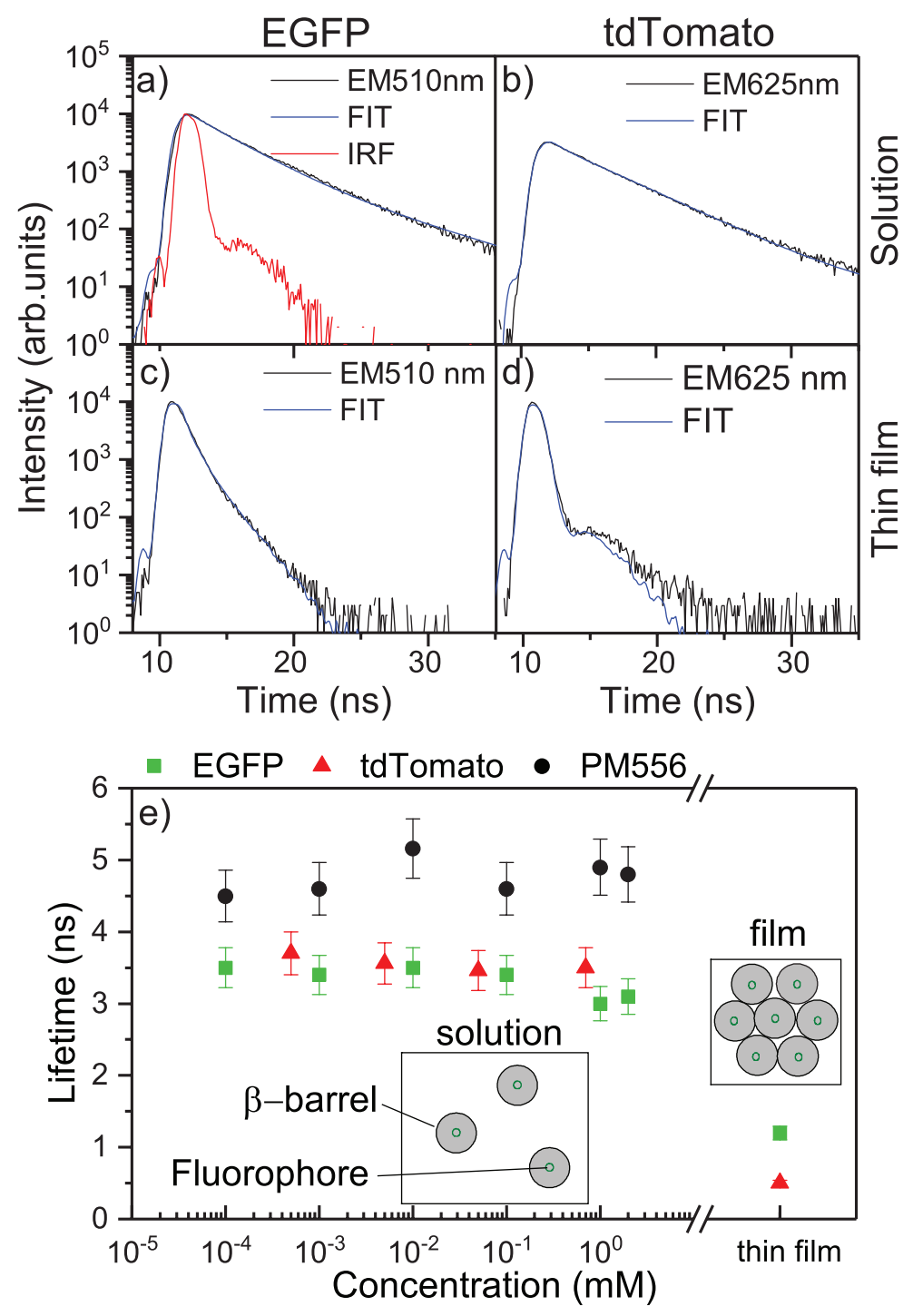

FIG. 3. Time-resolved PL decay for solutions and thin films of EGFP and tdTomato, measured using TCSPC. a) and c) Decay data for an EGFP solution (concentration, $1 \mathrm{mM}$ ) and a thin film of EGFP, respectively. b) and d) Same data for tdTomato. e) Average lifetimes for EGFP (green squares) and tdTomato (red triangles) for solutions of different concentration. For comparison, TCSPC lifetime measurements of the green-emitting synthetic pyrromethene dye PM556 are also shown (black circles). The insets give schematic illustrations of the arrangement of EGFP molecules in solution and thin film. 

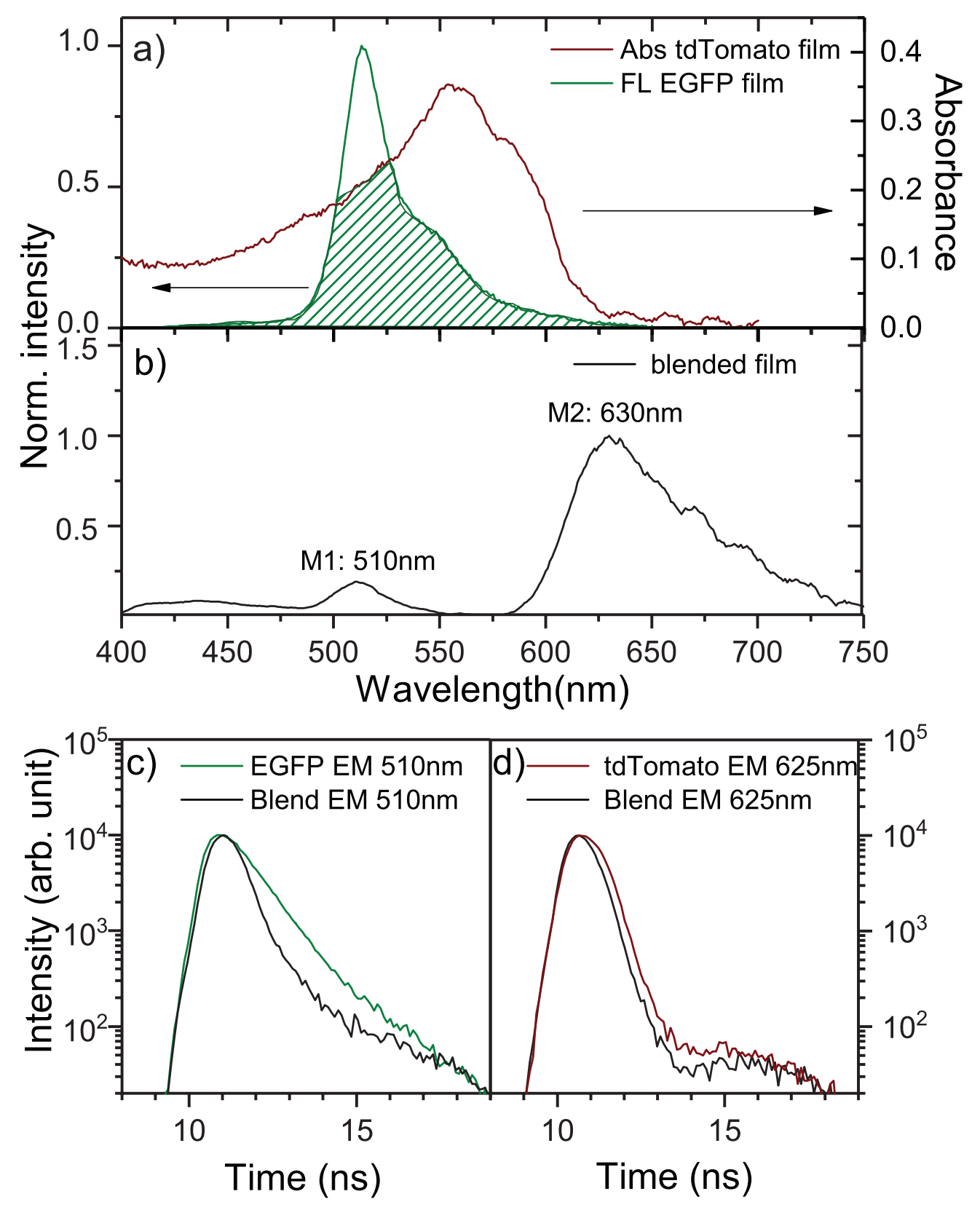

FIG. 4. a) Emission of an EGFP thin film and absorption of a tdTomato thin film. The dashed region marks the region of spectral overlap between EGFP emission and tdTomato absorption. b) PL of a thin film made of a blend of EGFP and tdTomato (7:3 by weight) with two emission maxima at $510 \mathrm{~nm}$ and $630 \mathrm{~nm}$. Excitation wavelength, $380 \mathrm{~nm}$. c) and d) TCSPC data for thin films of pure EGFP and pure tdTomato and for thin films of the 7:3 blend, recorded for emission at 510 and $625 \mathrm{~nm}$. 
a)
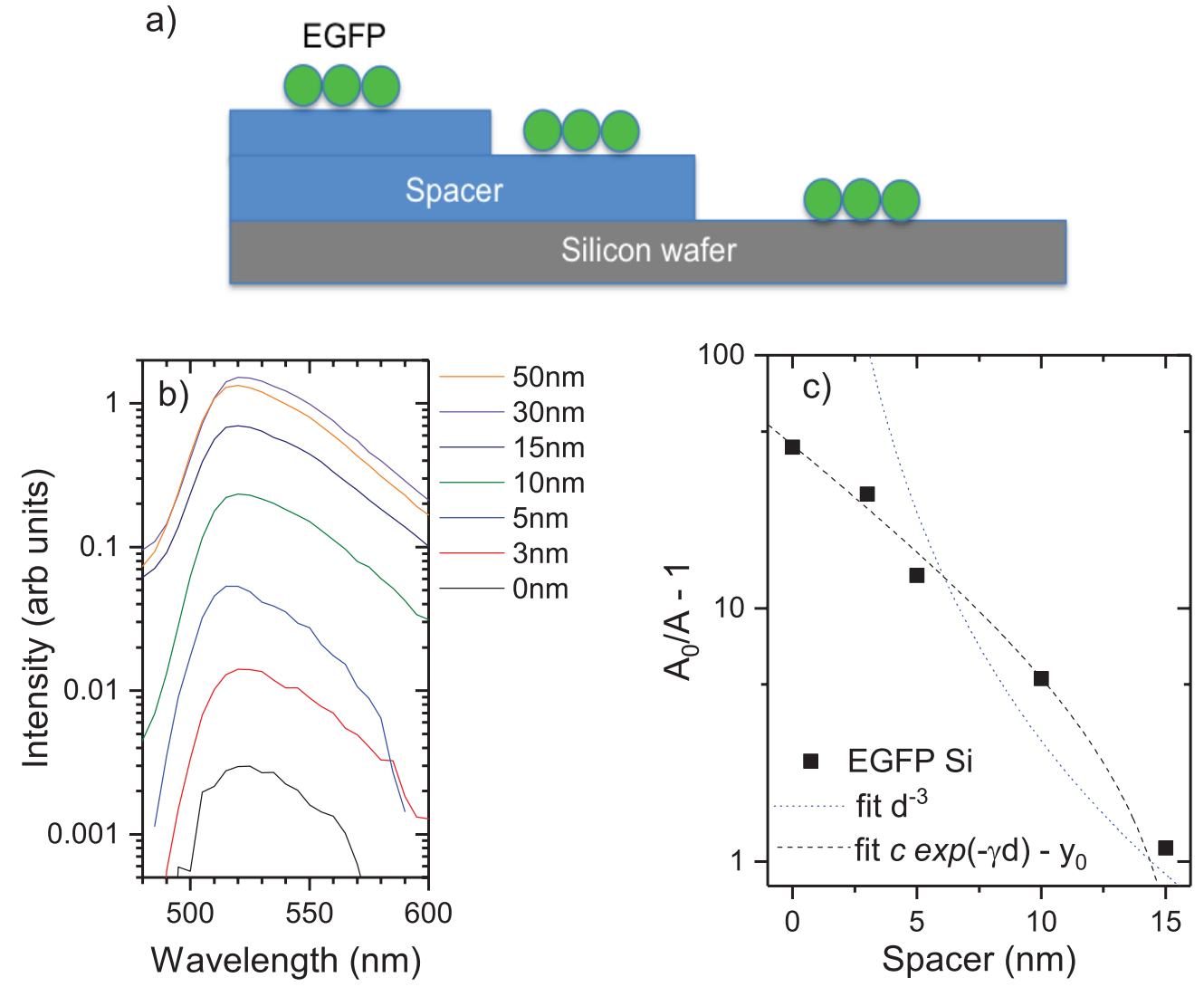

FIG. 5. a) A sketch of the investigated sample structure, with a thin EGFP layer (green circles) on top of a silicon wafer with a spacer layer of $\mathrm{Al}_{2} \mathrm{O}_{3}$ in between. The spacer layer thickness was varied between $d=0$ and $30 \mathrm{~nm}$. b) Intensity of fluorescence (on a logarithmic scale) versus wavelength for different spacer layer thicknesses. c) Change in quenching of EGFP emission as function of spacer layer thickness. See text for definition of $\left(A_{0} / A-1\right)$. Fits to the expected $d^{-3}$ behaviour and to an exponential decay function are also shown. 